\title{
In-Hospital and Long-Term Outcomes of Infective Endocarditis in Chronic Dialysis Patients
}

This article was published in the following Dove Press journal:

International Journal of General Medicine

\author{
Shuh-Kuan Liau' \\ George Kuo (D) \\ Chao-Yu Chen' \\ Yu-Cheng Chen ${ }^{2}$ \\ Yueh-An Lu' \\ Yu-Jr Lin (D) ${ }^{3}$ \\ Cheng-Chieh Hung' \\ Ya-Chung Tian' \\ Hsiang-Hao Hsu (D) \\ 'Department of Nephrology, Kidney \\ Research Center, Chang Gung Memorial \\ Hospital, Linkou Branch, College of \\ Medicine, Chang Gung University, \\ Taoyuan, Taiwan; ${ }^{2}$ Department of \\ Nephrology, Kidney Research Center, \\ Chang Gung Memorial Hospital, Linkou \\ Branch, Taoyuan, Taiwan; ${ }^{3}$ Research \\ Services Center for Health Information \\ from Chang Gung University, Taoyuan, \\ Taiwan
}

Purpose: To elucidate the in-hospital and long-term outcomes of infective endocarditis (IE) in end-stage kidney disease (ESKD) patients on chronic dialysis and to analyze the risk factors of mortality.

Patients and Methods: The case files of 1,817 patients who were hospitalized for IE over a 14-year period were retrospectively reviewed. Of these, 116 ESKD patients on chronic dialysis were enrolled in this study. Cox's proportional hazard model was used to evaluate the risk factors of mortality and long-term outcomes.

Results: The in-hospital mortality rate of the 116 enrolled patients was as high as $43.1 \%$. Patients who survived the index admission had a three-year mortality rate of $33 \%$. Univariate analysis was used to compare survivors and non-survivors; poor in-hospital outcomes were associated with the use of a tunneled cuffed catheter for dialysis access, a shorter duration hospitalization, shock or respiratory failure during hospitalization, a higher white blood count, a higher percentage of polymorphonuclear leukocytes, a higher C-reactive protein level, a lower serum albumin level, and a higher total bilirubin level. Following multivariate adjustment, shock (odds ratio, 9.29, with a $95 \%$ confidence interval [CI] of 2.78 to $34.24 ; p<0.001$ ) or respiratory failure (odds ratio, 25.16, with a 95\% CI of 5.63 to $153.54 ; p<0.001)$ during hospitalization was strongly associated with increased in-hospital mortality. Patients who underwent cardiac operations (odds ratio, 0.22 , with a $95 \%$ CI of 0.052 to $0.86 ; p=0.031$ ) had better in-hospital outcomes. Heart failure reduced ejection fraction (HFrEF) at the time of initial hospitalization was an independent risk factor for 3-year mortality (hazard ratio, 3.48, with a 95\% CI of 1.09 to 11.09; $p=0.035)$.

Conclusion: The outcomes of IE for ESKD patients on chronic dialysis were poor. Only $56.9 \%$ of these patients survived the index admission and their mortality rate over three years was $33 \%$. Shock or respiratory failure during hospitalization was associated with increased in-hospital mortality. Patients who underwent cardiac operations had better in-hospital outcomes. HFrEF at the time of initial hospitalization was an independent risk factor for threeyear mortality.

Keywords: infective endocarditis, chronic dialysis, end-stage kidney disease, blood-stream infection, infectious disease, sepsis

\section{Introduction}

Infective endocarditis (IE) in end-stage renal kidney disease (ESKD) patients on chronic dialysis has been shown to be significantly more common and to cause greater morbidity and mortality than in the general population. ${ }^{1-4}$ Previous investigations have reported in-hospital mortality rates and one-year mortality rates as high as $16.7 \%-28.8 \%{ }^{4-7}$ and $54.1 \%-65 \%,^{7-9}$ respectively. However, reports of
Correspondence: Hsiang-Hao Hsu Research Center, Chang Gung Memorial Hospital, Linkou Branch, College of Medicine, Chang Gung University, No. 5 Fu-Shin Street, Kweishan, Taoyuan, 333 Taiwan

Tel +886-3-328- 1200 ext. 8I8I

Fax +886-3-3282173

Email hsianghao@gmail.com
International Journal of General Medicine 2021:14 425-434 
prognostic factors are limited and patients' long-term outcomes have not been well described.

Maraj et al analyzed 32 IE patients who underwent hemodialysis and found a poor one-year prognosis that was related to a low hemoglobin level, an elevated leukocyte count, hypoalbuminemia, severe aortic and mitral regurgitation, and annular calcification in mitral valve IE. ${ }^{8}$ Shroff et al analyzed a total of 13,130 dialysis patients with bacterial endocarditis and found that the most powerful independent predictors of all-cause death were age, diabetes as cause of ESKD, and cerebrovascular accident or transient ischemic attack as a comorbid condition. ${ }^{7}$ Nori et al compared survivors and non-survivors among 52 patients with 54 episodes of IE in a predominantly African-American dialysis population, and found that MV disease and septic embolism were associated with inhospital mortality and that MV involvement and IE that is related to drug-resistant organisms are associated with poor outcomes following discharge from hospital. ${ }^{10}$ Although several prognostic factors had been proposed, the most powerful predictors of in-hospital mortality and long-term prognostic factors have not yet been found. The effect of heart failure, as the most important complication of IE, ${ }^{11,12}$ on the long-term survival of chronic dialysis patients with IE has not been elucidated.

This retrospective study attempted to identify the most important prognostic factors of IE for chronic dialysis patients, so that more aggressive therapy can be initiated early on for this targeted population and their poor prognosis improved.

\section{Patients and Methods}

\section{Patient Selection}

The medical records of chronic dialysis patients who had been admitted to a tertiary medical center for IE between January 2002 and December 2015 were retrospectively reviewed. The diagnosis of IE was defined using the International Classification of Diseases, Ninth Revision, Clinical Modification (ICD-9-CM) code 4210 during the index admission. A total of 1,817 IE patients were identified over a 14 -year period. The modified Duke criteria were applied in the diagnosis of IE. Of these patients, 116 had ESKD and required chronic dialysis and were therefore enrolled in this study. The study was approved by the institutional review board of Chang Gung Medical Foundation (approval number: 201600974B0), which waived the requirement for written informed consent from each participant because personal information was anonymized for this study. This study was conducted in accordance with the Declaration of Helsinki.

\section{Patients' Characteristics and Outcomes}

Baseline characteristics of sex, age, body weight/height, ESKD-associated comorbidities (diabetes mellitus, hypertension, coronary artery disease, heart failure, atrial fibrillation, history of prior stroke, peripheral artery disease, cirrhosis, peptic ulcer, chronic obstructive pulmonary disease, asthma, malignancy, immunosuppressive status), preIE valvular disease, history of cardiac surgery, and vascular access, were retrieved. Each patient's duration of hospitalization and complications during admission (shock, new stroke, respiratory failure) were documented. The results of blood examinations upon admission and on day seven of hospitalization were recorded. The pathogen of IE was identified by blood culture. Each patient was followed-up for three years from admission or until death.

\section{Statistical Analysis}

This investigation was a retrospective cohort study. Demographic data and clinical information are presented as mean \pm standard deviation (SD) and count (\%) for categorical data. The $t$-test and Chi-squared were used to compare continuous or categorical variables for the surviving and non-surviving groups. In multivariate analysis, logistic regression was used to assess the risk of inhospital death, and Cox regression analysis was used to evaluate the three-year survival rate. Variables that were determined to be significant in the univariate study were calculated. Kaplan-Meier three-year survival curves were plotted for groups with or without heart failure reduced ejection fraction (HFrEF). R statistical analysis software (copyright The $\mathrm{R}$ foundation for Statistical Computing) was used. All reported $p$-values were two-sided, and $p<0.05$ was considered to indicate statistical significance.

\section{Results}

\section{Demographic Data and Outcomes for Chronic Dialysis Patients with IE}

Of 1,817 patients who were hospitalized with IE from January 2002 to December 2015, 116 who underwent chronic dialysis were identified and enrolled in this study. Their mean age was $64.2 \pm 13.7$ years and their male-to-female ratio was 1:1.19 (Table 1). The distributions of age and sex did not differ between survivors 
Table I Demographic Data for Chronic Dialysis Patients with Infective Endocarditis

\begin{tabular}{|c|c|c|c|c|}
\hline \multirow[t]{2}{*}{ Variables } & Total $(\mathrm{N}=1 \mid 6)$ & Survived $(\mathrm{N}=66)$ & Died $(\mathbf{N}=50)$ & \multirow[t]{2}{*}{$p$ value } \\
\hline & \multicolumn{3}{|c|}{ No. (\%) } & \\
\hline Age (years) (mean \pm SD) & $64.2 \pm 13.7$ & $62.3 \pm 14.0$ & $66.7 \pm 13.0$ & 0.084 \\
\hline $\begin{array}{c}\text { Gender } \\
\text { Male } \\
\text { Female }\end{array}$ & $\begin{array}{l}53(45.7) \\
63(54.3)\end{array}$ & $\begin{array}{l}31(47.0) \\
35(53.0)\end{array}$ & $\begin{array}{l}22(44.0) \\
28(56.0)\end{array}$ & 0.897 \\
\hline \multicolumn{5}{|l|}{ Comorbidities } \\
\hline Diabetes mellitus & $63(54.3)$ & $39(59.1)$ & $24(48.0)$ & 0.318 \\
\hline Hypertension & $87(75.0)$ & $53(80.3)$ & $34(68.0)$ & 0.194 \\
\hline Coronary artery disease & $27(23.3)$ & $18(27.3)$ & $9(18.0)$ & 0.343 \\
\hline Heart failure & $37(31.9)$ & $18(27.3)$ & $19(38.0)$ & 0.305 \\
\hline Atrial fibrillation & $26(22.4)$ & $13(19.7)$ & $13(26.0)$ & 0.561 \\
\hline Prior stroke & $37(31.9)$ & $17(25.8)$ & $20(40.0)$ & 0.153 \\
\hline Peripheral arterial occlusive disease & $16(13.8)$ & $8(12.1)$ & $8(16.0)$ & 0.743 \\
\hline Cirrhosis & $12(10.3)$ & $10(15.2)$ & $2(4.0)$ & 0.100 \\
\hline Peptic ulcer & $42(36.2)$ & $28(42.4)$ & $14(28.0)$ & 0.160 \\
\hline Chronic obstructive pulmonary disease & $2(1.7)$ & $\mathrm{I}(\mathrm{I} .5)$ & $\mathrm{I}(2.0)$ & 1.000 \\
\hline Asthma & $3(2.6)$ & $2(3.0)$ & I (2.0) & 1.000 \\
\hline Malignancy & $13(11.2)$ & $7(10.6)$ & $6(12.0)$ & 1.000 \\
\hline Immunosuppressive status & $5(4.3)$ & $4(6.1)$ & I (2.0) & 0.545 \\
\hline \multicolumn{5}{|l|}{ Pre-IE valvular disease } \\
\hline Mitral valve & $28(24.1)$ & $16(24.2)$ & $12(24.0)$ & 1.000 \\
\hline Aortic valve & $14(12.1)$ & $6(9.1)$ & $8(16.0)$ & 0.399 \\
\hline Tricuspid valve & $18(15.5)$ & $7(10.6)$ & II (22.0) & 0.156 \\
\hline Pulmonary valve & $\mathrm{I}(0.9)$ & $\mathrm{I}(\mathrm{I} .5)$ & $0(0.0)$ & 1.000 \\
\hline Previous cardiac surgery & $9(7.8)$ & $5(7.6)$ & $4(8.0)$ & 1.000 \\
\hline \multicolumn{5}{|l|}{ Dialysis access } \\
\hline Tunneled cuffed catheter & $4 \mid(35.3)$ & $16(24.2)$ & $25(50.0)$ & $0.007^{*}$ \\
\hline Peritoneal dialysis & $5(4.3)$ & $4(6.1)$ & I (2.0) & 0.545 \\
\hline
\end{tabular}

Note: ${ }^{*} p$ value $<0.05$.

Abbreviation: SD, standard deviation.

and non-survivors. The number of patients who survived hospitalization was $66(56.9 \%)$ and the number who did not was 50 (43.1\%). Patients who survived index admission had a three-year mortality rate of 33\% (22 over 66). The average age of those who survived hospitalization was $62.3 \pm 14.0$ and that of those who did not was $66.7 \pm 13.0(p=0.084)$. Of all patients with IE, $75.0 \%$ had hypertension, $54.3 \%$ had diabetes, $36.2 \%$ had peptic ulcer, $31.9 \%$ had heart failure, $31.9 \%$ had suffered a prior stroke, $23.3 \%$ had coronary artery disease, $22.4 \%$ had atrial fibrillation, $13.8 \%$ had peripheral artery occlusive disease, $11.2 \%$ had a malignancy, $10.3 \%$ had cirrhosis, $4.3 \%$ had an immunosuppressive status, $2.6 \%$ had asthma, and $1.7 \%$ had chronic obstructive airway disease. Hypertension and diabetes were the two most common co-morbidities.

No significant difference in baseline comorbidities existed between in-hospital survivors and non-survivors. Mitral valve was the most common pre-IE valvular disease, present in $24.1 \%$ of patients, and nine patients (7.8\%) had had a previous cardiac surgery before IE was diagnosed. The type of previous cardiac surgery included 6 mitral valve replacements, 2 mitral valve repairs, 2 coronary artery bypass surgeries, and 1 aortic valve replacement. A tunneled cuffed catheter for dialysis access was used in $35.3 \%$ of patients and its frequency of use differed significantly between in-hospital survivors $(27.1 \%, \mathrm{n}=16)$ and non-survivors $(50.0 \%, \mathrm{n}=25), p=0.007$, based on 
a univariate analysis. Five (4.3\%) patients underwent peritoneal dialysis.

\section{Analysis of Clinical Characteristics of Chronic Dialysis Patients with IE}

According to univariate analysis, the duration of hospitalization was significantly longer for in-hospital survivors (49.9 days \pm 36.0 ) than non-survivors (34.5 days \pm 27.1 ), $p=0.013$ (Table 2). The frequencies of shock ( $18.2 \%$ survivors vs $84.0 \%$ non-survivors, $p<0.001)$ and respiratory failure $\quad(33.3 \%$ survivors vs $94.0 \%$ non-survivors, $p<0.0001)$ also significantly differed between the two groups. Patients' hemogram and biochemical data on days one and seven of hospitalization were recorded. On the first day of admission, the white blood count (WBC) was significantly higher $(12.25 \pm 5.88$ vs $15.01 \pm 6.31 \mathrm{k} / \mu \mathrm{L}$, $p=0.017)$ and the serum albumin level significantly lower $(3.05 \pm 0.48$ vs $2.63 \pm 0.49 \mathrm{~g} / \mathrm{dL}, p<0.001)$ in non-survivors. In day seven of hospitalization, a higher WBC (11.32 \pm 7.02 vs $15.54 \pm 8.42 \mathrm{k} / \mu \mathrm{L}, p=0.005)$, a higher percentage of polymorphonuclear leukocytes (PMN) $(77.59 \pm 11.90$ vs $83.24 \pm 8.26 \%, p=0.006)$, a lower percentage of lymphocytes $(13.42 \pm 7.87$ vs $8.59 \pm 5.86 \%, p=0.001)$, a higher C-reactive protein (CRP) level $(81.16 \pm 76.95$ vs 118.05 $\pm 76.09 \mathrm{mg} / \mathrm{L}, p=0.034)$, a lower serum albumin level $(2.99 \pm 0.53$ vs $2.63 \pm 0.58 \mathrm{~g} / \mathrm{dL}, p=0.015)$, and a higher total bilirubin level $(0.58 \pm 0.63$ vs $2.49 \pm 5.13 \mathrm{mg} / \mathrm{dL}$, $p=0.040$ ) were associated with higher in-hospital mortality.

Reduced ejection fraction (EF), defined as EF determined by echocardiography less than $50 \%$ at the time of initial hospitalization, was not common in either groups and the EF did not differ significantly between survivors and non-survivors. The mitral valve $(64.7 \%)$ was the most frequently infected valve, followed by the aortic valve (24.1\%). The type of valve infected did not differ significantly between survivors and non-survivors. Twenty-nine patients (25.0\%) received a cardiac operation. According to univariate analysis, receiving a cardiac operation and the valve operated upon were not significantly associated with mortality, but the protection afforded by the operation may have been masked or confounded by other risk factors, such as septic shock or respiratory failure. The pathogen of IE was identified by blood culture. Methicillin-resistant staphylococcus aureus (MRSA) was the most common pathogen (22.4\%). Polymicrobial (19.8\%), Methicillin-sensitive staphylococcus aureus (12.9\%), enterococcus faecalis (8.6\%), coagulase-negative staphylococcus (7.8\%), streptococcus
(4.3\%), gram-negative species $(2.6 \%)$, and gram-positive bacilli $(0.9 \%)$ were also identified. No pathogen was identified in $20.7 \%$ of the patients. The pathogen identified did not differ significantly between both groups.

\section{Multivariate Risk Factor Analysis of In-Hospital and Three-Year Mortality for Chronic Dialysis Patients with IE}

Logistic regression and Cox's proportional hazards model were used to identify important in-hospital and three-year prognostic factors (Table 3). Our results demonstrated that shock (odds ratio, 9.29, with a 95\% confidence interval [CI] of 2.78 to $34.24 ; p<0.001$ ) or respiratory failure (odds ratio, 25.16 , with a $95 \% \mathrm{CI}$ of 5.63 to $153.54 ; p<0.001$ ) during hospitalization was correlated with in-hospital mortality. Patients who received a cardiac operation had better in-hospital outcomes (odds ratio, 0.22 , with a $95 \%$ CI of 0.052 to $0.86 ; p=0.031$ ). Use of a tunneled cuffed catheter for dialysis access was associated with in-hospital mortality according to univariate analysis, but the association was insignificant following multivariate adjustment. The prognostic factors for three-year mortality of the patients who survived index hospitalization were identified. HFrEF, defined as left ventricular ejection fraction less than $50 \%$ at the time of initial hospitalization, was significantly correlated with higher three-year mortality (hazard ratio, 3.48, with a $95 \%$ CI of 1.09 to 11.09 ; $p=0.035$ ) (Figure 1).

\section{Discussion}

This retrospective study assessed the difference between survivors and non-survivors among IE patients who underwent chronic dialysis in terms of in-hospital and three-year mortality, previous reports of which are limited. Univariate analysis revealed that the use of a tunneled cuffed catheter for dialysis access, a shorter hospital duration, shock, respiratory failure during hospitalization, and worse hemogram and biochemistry data one week after admission were significantly associated with a poorer in-hospital prognosis. No difference in age, gender or baseline comorbidities existed between survivors and non-survivors. According to multivariate analysis, with respect to short-term prognosis, shock or respiratory failure in the hospital was associated with higher in-hospital mortality. Patients who underwent an operation had better in-hospital outcomes. With respect to long-term prognosis, HFrEF at the time of initial hospitalization was related to higher three-year mortality. 
Table 2 Clinical Characteristics of Chronic Dialysis Patients with Infective Endocarditis

\begin{tabular}{|c|c|c|c|c|}
\hline \multirow[t]{2}{*}{ Characteristics } & Total $(\mathrm{N}=1 \mid 6)$ & Survived $(\mathrm{N}=66)$ & Died $(\mathbf{N}=\mathbf{5 0})$ & \multirow[t]{2}{*}{$p$ value } \\
\hline & \multicolumn{3}{|c|}{ No. (\%) } & \\
\hline Hospital stay (days) (mean \pm SD) & $43.3 \pm 33.2$ & $49.9 \pm 36.0$ & $34.5 \pm 27.1$ & $0.013 *$ \\
\hline \multicolumn{5}{|l|}{ Complications } \\
\hline Shock & $54(46.6)$ & $12(18.2)$ & $42(84.0)$ & $<0.00 I^{*}$ \\
\hline Stroke & $27(23.3)$ & $16(24.2)$ & II (22.0) & 0.951 \\
\hline Respiratory failure & $69(59.5)$ & $22(33.3)$ & $47(94.0)$ & $<0.00 I^{*}$ \\
\hline \multicolumn{5}{|l|}{ Laboratory data } \\
\hline \multicolumn{5}{|l|}{ Hospital Day I } \\
\hline WBC $(k / \mu L)$ & $13.44 \pm 6.20$ & $12.25 \pm 5.88$ & $|5.0| \pm 6.3 \mid$ & $0.017^{*}$ \\
\hline Hemoglobin (g/dL) & $9.16 \pm 1.89$ & $9.24 \pm 2.06$ & $9.04 \pm 1.62$ & 0.591 \\
\hline Platelet $(\mathrm{k} / \mu \mathrm{L})$ & $158.80 \pm 91.96$ & $154.98 \pm 82.87$ & $|63.84 \pm| 03.4 \mid$ & 0.610 \\
\hline PMN (\%) & $84.50 \pm 8.45$ & $83.91 \pm 8.80$ & $85.29 \pm 7.99$ & 0.388 \\
\hline Lymphocyte (\%) & $8.59 \pm 5.83$ & $8.96 \pm 5.61$ & $8.12 \pm 6.14$ & 0.446 \\
\hline CRP (mg/L) & $134.86 \pm 90.69$ & $122.53 \pm 85.93$ & $152.75 \pm 95.45$ & 0.105 \\
\hline $\mathrm{ESR}(\mathrm{mm} / \mathrm{hr})$ & $71.08 \pm 39.79$ & $73.57 \pm 38.25$ & $65.62 \pm 43.74$ & 0.514 \\
\hline Albumin $(\mathrm{g} / \mathrm{dL})$ & $2.86 \pm 0.53$ & $3.05 \pm 0.48$ & $2.63 \pm 0.49$ & $<0.001 *$ \\
\hline Total bilirubin (mg/dL) & $0.88 \pm 0.80$ & $0.83 \pm 0.72$ & $0.93 \pm 0.90$ & 0.546 \\
\hline \multicolumn{5}{|l|}{ Hospital Day 7} \\
\hline WBC $(\mathrm{k} / \mu \mathrm{L})$ & $13.22 \pm 7.93$ & $11.32 \pm 7.02$ & $15.54 \pm 8.42$ & $0.005^{*}$ \\
\hline Hemoglobin $(g / d L)$ & $8.98 \pm 1.60$ & $9.08 \pm 1.56$ & $8.85 \pm 1.67$ & 0.480 \\
\hline Platelet $(\mathrm{k} / \mu \mathrm{L})$ & $165.64 \pm|10.9|$ & $171.47 \pm 107.26$ & $|58.6| \pm|| 5.88$ & 0.551 \\
\hline PMN (\%) & $80.13 \pm 10.76$ & $77.59 \pm 11.90$ & $83.24 \pm 8.26$ & $0.006 *$ \\
\hline Lymphocyte (\%) & $11.26 \pm 7.42$ & $13.42 \pm 7.87$ & $8.59 \pm 5.86$ & $0.001 *$ \\
\hline CRP (mg/L) & $96.27 \pm 78.29$ & $81.16 \pm 76.95$ & $118.05 \pm 76.09$ & $0.034^{*}$ \\
\hline $\mathrm{ESR}(\mathrm{mm} / \mathrm{hr})$ & $81.83 \pm 42.13$ & $75.50 \pm 39.96$ & $95.89 \pm 45.82$ & 0.235 \\
\hline Albumin $(g / d L)$ & $2.82 \pm 0.58$ & $2.99 \pm 0.53$ & $2.63 \pm 0.58$ & $0.015^{*}$ \\
\hline Total bilirubin (mg/dL) & $1.49 \pm 3.66$ & $0.58 \pm 0.63$ & $2.49 \pm 5.13$ & $0.040 *$ \\
\hline \multicolumn{5}{|l|}{ Echocardiography in hospital } \\
\hline LVEF & $0.64 \pm 0.14$ & $0.66 \pm 0.13$ & $0.62 \pm 0.15$ & 0.157 \\
\hline LVEDD (mm) & $48.88 \pm 8.52$ & $49.55 \pm 8.19$ & $47.96 \pm 8.96$ & 0.326 \\
\hline IVS (mm) & $12.69 \pm 2.90$ & $|3.00 \pm 2.8|$ & $12.27 \pm 3.00$ & 0.187 \\
\hline LVPW (mm) & $11.68 \pm 3.60$ & $11.42 \pm 2.58$ & $12.02 \pm 4.67$ & 0.385 \\
\hline $\mathrm{LV}$ mass (gm) & $238.70 \pm 99.14$ & $243.45 \pm 84.86$ & $232.18 \pm 116.61$ & 0.551 \\
\hline
\end{tabular}


Table 2 (Continued).

\begin{tabular}{|c|c|c|c|c|}
\hline \multirow[t]{2}{*}{ Characteristics } & Total $(\mathrm{N}=1 \mid 6)$ & Survived $(N=66)$ & Died $(\mathrm{N}=50)$ & \multirow[t]{2}{*}{$p$ value } \\
\hline & \multicolumn{3}{|c|}{ No. (\%) } & \\
\hline $\mathrm{LV}$ mass index $\left(\mathrm{gm} / \mathrm{m}^{2}\right)$ & $151.58 \pm 68.14$ & $151.33 \pm 54.33$ & $151.98 \pm 85.77$ & 0.962 \\
\hline \multicolumn{5}{|l|}{ Valve infected } \\
\hline Mitral valve & $75(64.7)$ & $43(65.2)$ & $32(64.0)$ & 1.000 \\
\hline Aortic valve & $28(24.1)$ & II (16.7) & $17(34.0)$ & 0.052 \\
\hline Mitral and aortic valve & $9(7.8)$ & $3(4.5)$ & $6(12.0)$ & 0.256 \\
\hline Tricuspid valve & II (9.5) & $5(7.6)$ & $6(12.0)$ & 0.627 \\
\hline Pulmonary valve & I (0.9) & $\mathrm{I}(\mathrm{I} .5)$ & $0(0.0)$ & 1.000 \\
\hline Other sites & $3(2.6)$ & $2(3.0)$ & I (2.0) & 1.000 \\
\hline No vegetation & $9(7.8)$ & $7(10.6)$ & $2(4.0)$ & 0.334 \\
\hline Received operation & $29(25.0)$ & $19(28.8)$ & $10(20.0)$ & 0.387 \\
\hline \multicolumn{5}{|l|}{ Valve of operation } \\
\hline Mitral valve & $21(18.1)$ & $14(2 \mid .2)$ & $7(14.0)$ & 0.450 \\
\hline Aortic valve & II (9.5) & $7(10.6)$ & $4(8.0)$ & 0.877 \\
\hline Mitral and aortic valve & $7(6.0)$ & $4(6.1)$ & $3(6.0)$ & 1.000 \\
\hline Tricuspid valve & $6(5.2)$ & $3(4.5)$ & $3(6.0)$ & 1.000 \\
\hline Pulmonary valve & $0(0.0)$ & $0(0.0)$ & $0(0.0)$ & NA \\
\hline \multicolumn{4}{|l|}{ Blood culture } & 0.476 \\
\hline MRSA & $26(22.4)$ & $13(19.7)$ & $13(26.0)$ & \\
\hline MSSA & $15(12.9)$ & $8(12.1)$ & $7(14.0)$ & \\
\hline Coagulase-negative Staphylococcus & $9(7.8)$ & $4(6.1)$ & $5(10.0)$ & \\
\hline Enterococcus & $10(8.6)$ & $6(9.1)$ & $4(8.0)$ & \\
\hline Streptococcus species & $5(4.3)$ & $4(6.0)$ & I (2.0) & \\
\hline Gram-negative species & $3(2.6)$ & $2(3.0)$ & I (2.0) & \\
\hline Gram-positive bacilli & I $(0.9)$ & $\mathrm{I}(\mathrm{I} .5)$ & $0(0.0)$ & \\
\hline Polymicrobial & $23(19.8)$ & $10(15.2)$ & $13(26.0)$ & \\
\hline Culture-negative & $24(20.7)$ & $18(27.3)$ & $6(12.0)$ & \\
\hline
\end{tabular}

Note: $* p$ value $<0.05$.

Abbreviations: SD, standard deviation; WBC, white blood count; PMN, polymorphonuclear leukocytes; CRP, C-reactive protein; ESR, erythrocyte sedimentation rate; LVEF, left ventricular ejection fraction; LVEDD, left ventricular end-diastolic diameter; IVS, interventricular septum; IVPW, left ventricle posterior wall; MSSA, methicillinsusceptible Staphylococcus aureus; MRSA, methicillin-resistance Staphylococcus aureus.

The relevant literature reports in-hospital mortality rates of $16.7 \%-28.8 \% ;^{4-7}$ a higher in-hospital mortality rate $(43.1 \%)$ was observed in this study. The greater prevalence of diabetes mellitus, older age, and poorer nutrition of the patients in the present study population may explain this difference. We found shorter 


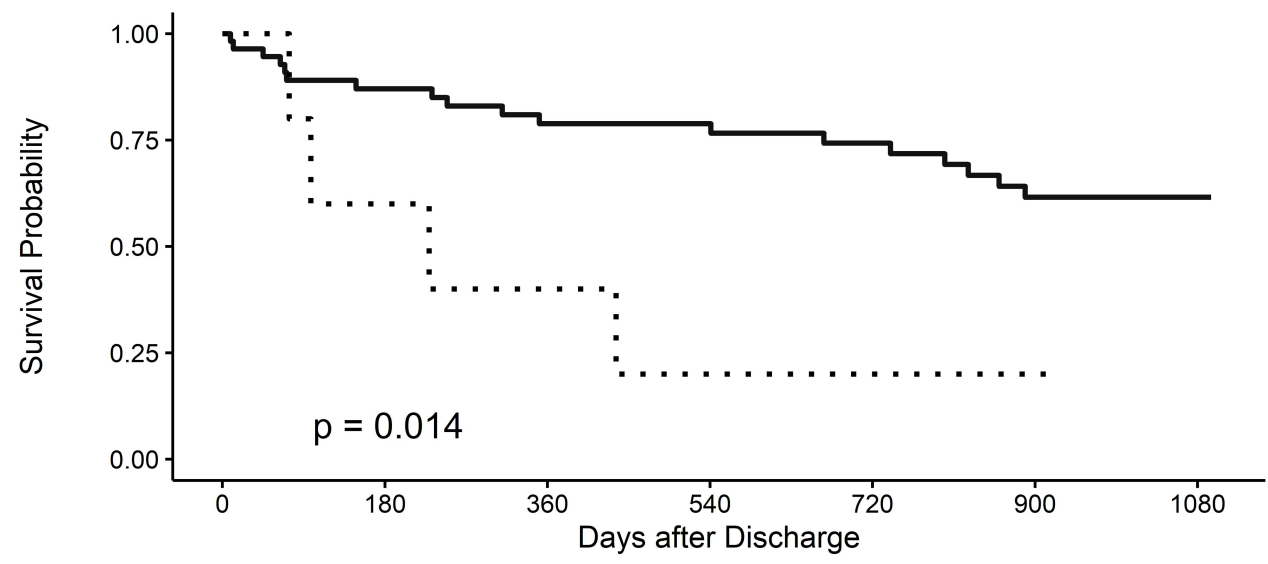

Number at risk

\begin{tabular}{|c|c|c|c|c|c|c|}
\hline No HFrEF & 60 & 43 & 38 & 35 & 30 & 24 \\
\hline HFrEF & 6 & 3 & 2 & 1 & 1 & 1 \\
\hline
\end{tabular}

Figure I Kaplan-Meier plot for three-year survival with and without heart failure reduced ejection fraction.

Abbreviation: HFrEF, heart failure reduced ejection fraction.

Table 3 Multivariate Analysis of Risk Factors for In-Hospital Mortality and Three-Year Mortality

\begin{tabular}{|l|l|r|}
\hline Population & Variable & Risk Measurement \\
\hline In-Hospital & & Odds Ratio (95\% CI) \\
& & \\
& Male & $0.55(0.15-1.87)$ \\
& Age & $1.03(0.98-1.08)$ \\
& Diabetes mellitus & $0.38(0.10-1.3 \mathrm{I})$ \\
& Shock & $9.29(2.78-34.24)^{*}$ \\
& Respiratory failure & $25.16(5.63-153.54)^{*}$ \\
& Operation & $0.22(0.052-0.86)^{*}$ \\
& Tunneled cuffed & $2.69(0.77-10.07)$ \\
& catheter & \\
& MRSA bacteremia & $0.71(0.16-2.96)$ \\
\hline \multirow{3}{*}{ Mortality } & & Hazard Ratio (95\% CI) \\
& & $1.81(0.73-4.50)$ \\
& Male & $1.03(0.99-1.06)$ \\
& Age & $0.62(0.25-1.55)$ \\
& Diabetes mellitus & $1.69(0.50-5.70)$ \\
& Shock & $2.13(0.62-7.34)$ \\
& Respiratory failure & $0.58(0.18-1.92)$ \\
& Operation & $1.37(0.51-3.72)$ \\
& Tunneled cuffed & $3.48(1.09-11.09)^{*}$ \\
\hline & catheter &
\end{tabular}

Note: $*_{p}$ value $<0.05$.

Abbreviations: $\mathrm{Cl}$, confidence interval; MRSA, methicillin-resistance Staphylococcus aureus; EF, ejection fraction. hospitalization was associated with a worse outcome, which is rather paradoxical, as patients with a favorable course of the disease would be expected to be discharged earlier. We suggested this finding is because these patients did not survive a longer hospital stay. Several studies have analyzed the mortality risk factors in chronic dialysis patients with IE. Shroff et al analyzed the cases of a total of 13,130 dialysis patients with bacterial endocarditis and found the most powerful independent predictors of allcause death were age, diabetes as a cause of ESKD, and cerebrovascular accident or transient ischemic attack as a comorbid condition. ${ }^{7}$ Nori et al compared survivors and non-survivors among 52 patients with 54 episodes of IE in a predominantly African-American dialysis population and found that MV disease and septic embolism were associated with in-hospital mortality; they also found that MV involvement and IE that is related to drug-resistant organisms were associated with poor outcomes following discharge from hospital. ${ }^{10}$ However, this study did not find a significant association between baseline comorbidity or involving microorganisms and in-hospital prognosis.

A higher IE incidence in ESKD patients than general populations may be related to the fact that hemodialysis patients require frequent arteriovenous fistula/graft puncturing. Patients with a central venous catheter for 
hemodialysis access also have a higher risk of endocarditis than those with other means of access. ${ }^{4}$ In this study, $35.3 \%$ of patients used a tunneled cuffed catheter for dialysis access. Univariate analysis revealed a poor inhospital prognosis in patients with a tunneled cuffed catheter for dialysis access, but after multivariate adjustment, this association was insignificant. The tunneled cuffed catheters were inserted prior to IE, which served as a possible route of bacteria entrance, ${ }^{13}$ and all these catheters were definitely removed at the time of IE diagnosis. The possible explanation for the relation between the use of the catheters and higher mortality was that patients who used the catheter as hemodialysis access were generally older and frailer, and usually experienced primary immaturity of the vascular access. These patients had more comorbidities as well. The use of catheters reflected the characteristics of this population. These findings suggest that the early removal of a tunnel cuffed catheter may reduce both the incidence of IE for chronic dialysis patients and the mortality rate.

Schoenberg et al found that the mortality rate of systemic inflammatory response syndrome (SIRS) ranged from $6 \%$ to $7 \%$ and that of septic shock exceeded $50 \%$ in an ICU population. ${ }^{14}$ Unsurprisingly, both univariate and multivariate analyses in this study revealed that shock during hospitalization, which was associated with more severe disease activity, was strongly associated with higher in-hospital mortality. However, shock was not associated with reduced three-year survival. Respiratory failure was strongly associated with worse in-hospital prognosis, but not associated with worse long-term survival. Using the National Inpatient Sample (NIS), Khalif et al retrospectively identified a total of 234,731 admissions with a primary diagnosis of IE from 1999 to 2014 and found that respiratory failure was present as a secondary diagnosis in $7.6 \%$ of the patients. They concluded that respiratory failure is an underappreciated complication of IE and is associated with a significant increase in in-hospital mortality, length of stay, and total charges. ${ }^{15}$ Based on these findings, we recommend more aggressive therapy for patients who develop shock or respiratory failure in hospital.

Maraj et al analyzed the cases of 32 IE patients who underwent hemodialysis and found that a poor one-year prognosis was associated with a low hemoglobin level, an elevated leukocyte count, hypoalbuminemia, severe aortic and mitral regurgitation, and annular calcification in mitral valve IE. ${ }^{8}$ In this study, as markers of inflammation, infection, and nutrition levels, a higher leukocyte count, a higher C-reactive protein level, a higher PMN, a lower albumin level, and a higher total bilirubin level after one week of hospitalization were associated with poor inhospital prognosis, but these associations were insignificant following multivariate adjustment. Studies have reported that the most commonly affected heart valve in hemodialysis patients with IE is the MV because this valve is more vulnerable to volume overload-related damage and calcifications than any other heart valve in these patients. ${ }^{16}$ This work is consistent with this finding as the MV (64.7\%) was the most commonly infected valve in chronic dialysis patients with IE, followed by the aortic valve (24.1\%). Nevertheless, the type of valve infected was not associated with mortality, as stated by our results.

The value of cardiac surgery for chronic dialysis patients with IE in ESKD is debatable. High operation mortality in cases of IE has been reported. Dohmen et al retrospectively collected 992 cases of aortic valve IE, diagnosed according to modified Duke criteria, in patients who received aortic valve surgery for IE; 45 of these patients received long-term hemodialysis preoperatively. The mortality within 30 days of surgery was as high as $42.2 \%{ }^{17}$ Chaudry et al retrospectively analyzed 190 Staphylococcus aureus endocarditis in hemodialysisdependent ESKD and found no one-year mortality difference between operated (50\%) and non-operated patients $(48.5 \%) .{ }^{18}$ Guo et al found a higher mortality within 30 days of surgery for IE in dialysis-dependent patients than those with normal renal function (14\% vs 5.8\%), and raised the question of whether indications for surgery in the general population are appropriate for patients who are dialysis-dependent. ${ }^{19}$ However, some studies have revealed the benefits of such operations. Raza et al found a five-year survival rate of $24 \%$ for patients with IE on hemodialysis and suggested surgical intervention for this high-risk population. ${ }^{20}$ A systemic review and metaanalysis of 32 studies that compared valve reoperation in cases of prosthetic valve IE and medical therapy concluded that surgery is associated with a lower 30-day mortality and greater survival at follow-up. ${ }^{21}$ In this study, 19 of $29(65.5 \%)$ patients who underwent such an operation survived. According to univariate analysis, the cardiac operation was not significantly associated with reduced mortality, but the protective role of the operation may have been masked or confounded by other risk factors, such as septic shock or respiratory failure. Following multivariate adjustment, patients who underwent an 
operation were found to have better in-hospital outcomes. This work demonstrates that a cardiac operation as aggressive management should be considered in IE patients with chronic dialysis.

Staphylococcus aureus has been reported to be the predominant causative pathogen of IE in chronic dialysis patients. $^{8,22}$ Consistent with this finding, $S$. aureus was the most common organism (35.3\%) in our study group. Moreover, MRSA endocarditis reportedly has higher mortality than endocarditis that is caused by most other microorganisms. Kuo et al retrospectively reviewed the cases of 22 patients with a definite diagnosis of IE that was caused by $S$. aureus and found a mortality rate of MRSA endocarditis in hemodialysis patients of $90 \%(9 / 10) .{ }^{23}$ Yoon et al compared the clinical features and mortality rates ofMRSA and MSSA strains of IE in 32 patients and found that MRSA IE (50\%) had a higher mortality rate than MSSA IE (9.1\%). ${ }^{24}$ However, both univariate and multivariate analyses did not reveal a significant association between the causative pathogen and the prognosis in our study group. High awareness of the likelihood of MRSA IE and the early empirical use of more "potent" antibiotics in our study population may explain the reduction of the variation among the mortality rates associated with MRSA and other organisms.

Heart failure is the most frequent complication of IE and the most common indication for surgery in IE. $^{25}$ Nadji et al, who analyzed 259 cases of definite left-side native valve IE, found that congestive heart failure is an independent predictor of in-hospital [OR 3.8 (1.7-9.0); $p=0.0013$ ] and one-year mortality [HR 1.8 (1.1-3.0); $p=0.007]$. They also found that in heart failure patients, early surgery is independently associated with reduced mortality and should be widely considered to improve outcomes. $^{26}$ In 2015, ESC guidelines for the management of IE suggested that unless severe co-morbidity is present, heart failure is an indication for early surgery in native valve IE and prosthetic valve IE - even in patients with cardiogenic shock. ${ }^{27}$ Based on a multivariate analysis, HFrEF at the time of initial hospitalization was not correlated with in-hospital mortality but was correlated with three-year mortality in the present work. This finding may favor cardiac operation, owing to its possible preservation of heart function, to improve long-term survival of chronic dialysis patients with IE. Although well-established indication of surgical intervention for $\mathrm{IE}^{27,28}$ the decision of cardiac operation in chronic dialysis patients is more complex. Woller Iii et al found nearly $25 \%$ of patients with left-sided IE had a Class I indication for surgery and were not offered early surgery, reflecting the complexity of caring for this population with multiple comorbidities and high acuity of illness. $^{29}$

This study has several limitations. First, this study was a retrospective study at a single medical center with predominantly Asian people so its findings may not apply to the general population. Second, since this study involved one center, the number of considered cases was limited, reducing the capacity to detect significance with respect to some variables. Third, only chronic dialysis patients were enrolled and the in-hospital and long-term outcomes of IE for chronic dialysis patients and non-dialysis patients were not compared. Further study is needed. Fourth, EF less than $50 \%$, which we used to define HFrEF, may not reflect heart failure due to acute valve regurgitation, which is mostly congestive in nature and not necessarily associated with a reduced EF. The true long-term prognosis of heart failure may be worse than our study results, because we did not count heart failure without reduced EF. Nevertheless, this work provides important information about prognostic factors of IE in chronic dialysis patients.

\section{Conclusion}

Despite advances in medical treatment, the outcomes of IE in ESKD patients who underwent chronic dialysis and were enrolled in this study were poor. Only $56.9 \%$ of these patients survived index hospitalization and their mortality rate over three years was $33 \%$. Shock or respiratory failure during hospitalization was associated with increased in-hospital mortality. Patients who underwent cardiac operations had better in-hospital outcomes. HFrEF at the time of initial hospitalization was an independent risk factor for three-year mortality. These factors may be used to identify ESKD patients with IE who require more aggressive treatments.

\section{Acknowledgments}

The authors would like to thank the Research Services Center for Health Information at Chang Gung University for conducting the statistical analysis and Linkou ChangGung Memorial Hospital for a Research Grant (CMRPG1J0031).

\section{Disclosure}

The authors report no conflicts of interest in this work. 


\section{References}

1. Nucifora G, Badano LP, Viale P, et al. Infective endocarditis in chronic haemodialysis patients: an increasing clinical challenge. Eur Heart J. 2007;28(19):2307-2312. doi:10.1093/eurheartj/ehm278

2. Abbott KC, Agodoa LY. Hospitalizations for bacterial endocarditis after initiation of chronic dialysis in the United States. Nephron. 2002;91(2):203-209. doi:10.1159/000058393

3. Strom BL, Abrutyn E, Berlin JA, et al. Risk factors for infective endocarditis: oral hygiene and nondental exposures. Circulation. 2000;102(23):2842-2848. doi:10.1161/01.CIR.102.23.2842

4. Chaudry MS, Carlson N, Gislason GH, et al. Risk of infective endocarditis in patients with end stage kidney disease. Chin $\mathrm{J} \mathrm{Am}$ Soc Nephrol. 2017;12:CJN.02320317. doi:10.2215/CJN.02320317

5. Bhatia N, Agrawal S, Garg A, et al. Trends and outcomes of infective endocarditis in patients on dialysis. Clin Cardiol. 2017;40 (7):423-429. doi:10.1002/clc.22688

6. Baroudi S, Qazi RA, Lentine KL, Bastani B. Infective endocarditis in haemodialysis patients: 16-year experience at one institution. NDT Plus. 2008;1(4):253-256. doi:10.1093/ndtplus/sfn026

7. Shroff GR, Herzog CA, Ma JZ, Collins AJ. Long-term survival of dialysis patients with bacterial endocarditis in the United States. Am J Kidney Dis. 2004;44(6):1077-1082. doi:10.1053/j.ajkd.2004.08.030

8. Maraj S, Jacobs LE, Kung SC, et al. Epidemiology and outcome of infective endocarditis in hemodialysis patients. Am J Med Sci. 2002;324(5):254-260. doi:10.1097/00000441-200211000-00004

9. McCarthy JT, Steckelberg JM. Infective endocarditis in patients receiving long-term hemodialysis. Mayo Clin Proc. 2000;75 (10):1008-1014. doi:10.4065/75.10.1008

10. Nori US, Manoharan A, Thornby JI, Yee J, Parasuraman R, Ramanathan V. Mortality risk factors in chronic haemodialysis patients with infective endocarditis. Nephrol Dialysis Transplant. 2006;21(April):2184-2190. doi:10.1093/ndt/gfl200

11. Mocchegiani R, Nataloni M. Complications of infective endocarditis. Cardiovasc Hematol Disord Drug Targets. 2009;9(4):240-248. doi:10.2174/1871529X10909040240

12. Rekik S, Trabelsi I, Hentati M, et al. Infective endocarditis in hemodialysis patients: clinical features, echocardiographic data and outcome: a 10-year descriptive analysis. Clin Exp Nephrol. 2009;13 (4):350-354. doi:10.1007/s10157-009-0172-8

13. Jeon HD, Lo KB, Quintero EE, et al. Dialysis access as a source of infective endocarditis in dialysis patients. Monaldi Arch Chest Dis. 2020;90(4). doi:10.4081/monaldi.2020.1505.

14. Schoenberg MH, Weiss M, Radermacher P. Outcome of patients with sepsis and septic shock after ICU treatment. Langenbecks Arch Surg. 1998;383(1):44-48. doi:10.1007/s004230050090

15. Khalif A, Pati P, Shanmugam B, Perimbeti S, Ward J. Respiratory failure in acute infective endocarditis, trends and outcomes: data from the national inpatient sample from 1999-2014. Chest. 2017;152:A63.

16. Hsiao CC, Weng CH, Li YJ, et al. Comparison of the clinical features and outcomes of infective endocarditis between hemodialysis and non-hemodialysis patients. Ther Clin Risk Manag. 2017;13:663-668. doi:10.2147/TCRM.S135262

International Journal of General Medicine

\section{Publish your work in this journal}

The International Journal of General Medicine is an international, peer-reviewed open-access journal that focuses on general and internal medicine, pathogenesis, epidemiology, diagnosis, monitoring and treatment protocols. The journal is characterized by the rapid reporting of reviews, original research and clinical studies
17. Dohmen PM, Binner C, Mende M, et al. Outcome of aortic valve replacement for active infective endocarditis in patients on chronic hemodialysis. Ann Thorac Surg. 2015;99(2):532-538. doi:10.1016/j. athoracsur.2014.08.025

18. Chaudry MS, Gislason GH, Kamper AL, et al. The impact of hemodialysis on mortality risk and cause of death in Staphylococcus aureus endocarditis. BMC Nephrol. 2018;19. doi:10.1186/s12882-018-1016-0

19. Guo M, St Pierre E, Clemence J, et al. Impact of chronic renal failure on surgical outcomes in patients with infective endocarditis. Ann Thorac Surg. 2020. doi:10.1016/j.athoracsur.2020.06.023

20. Raza S, Hussain ST, Rajeswaran J, et al. Value of surgery for infective endocarditis in dialysis patients. $J$ Thorac Cardiovasc Surg. 2017;154(1):61-70.e66. doi:10.1016/j.jtcvs.2017.02.063

21. Mihos CG, Capoulade R, Yucel E, Picard MH, Santana O. Surgical versus medical therapy for prosthetic valve endocarditis: a meta-analysis of 32 studies. Ann Thorac Surg. 2017;103 (3):991-1004. doi:10.1016/j.athoracsur.2016.09.083

22. Wang CY, Wang YC, Yang YS, et al. Microbiological features, clinical characteristics and outcomes of infective endocarditis in adults with and without hemodialysis: a 10-year retrospective study in Northern Taiwan. $J$ Microbiol Immunol Infect. 2020;53 (2):336-343. doi:10.1016/j.jmii.2018.08.013

23. Kuo CB, Lin JC, Peng MY, Wang NC, Chang FY. Endocarditis: impact of methicillin-resistant Staphylococcus aureus in hemodialysis patients and community-acquired infection. J Microbiol Immunol Infect. 2007;40(4):317-324.

24. Yoon HJ, Choi JY, Kim CO, Kim JM, Song YGA. Comparison of clinical features and mortality among methicillin-resistant and methicillin-sensitive strains of Staphylococcus aureus endocarditis. Yonsei Med J. 2005;46(4):496-502. doi:10.3349/ymj.2005.46.4.496

25. Tornos P, Iung B, Permanyer-Miralda G, et al. Infective endocarditis in Europe: lessons from the Euro heart survey. Heart. 2005;91 (5):571-575. doi:10.1136/hrt.2003.032128

26. Nadji G, Rusinaru D, Rémadi JP, Jeu A, Sorel C, Tribouilloy C. Heart failure in left-sided native valve infective endocarditis: characteristics, prognosis, and results of surgical treatment. Eur J Heart Fail. 2009;11(7):668-675. doi:10.1093/eurjhf/hfp077

27. Habib G, Lancellotti P, Antunes MJ, et al. 2015 ESC Guidelines for the management of infective endocarditis: The Task Force for the Management of Infective Endocarditis of the European Society of Cardiology (ESC) Endorsed by: European Association for Cardio-Thoracic Surgery (EACTS), the European Association of Nuclear Medicine (EANM). Eur Heart J. 2015;36(44):3075-3128. doi:10.1093/eurheartj/ehv319

28. Baddour LM, Wilson WR, Bayer AS, et al. Infective endocarditis in adults: diagnosis, antimicrobial therapy, and management of complications: a scientific statement for healthcare professionals from the American Heart Association. Circulation. 2015;132:1435-1486. doi:10.1161/CIR.0000000000000296

29. Woller Iii JA, Walsh VL, Robichaux C, Thourani VH, Jacob JT. Predictors of surgical intervention in dialysis patients with infective endocarditis. Open Forum Infect Dis. 2018;5(11):ofy265. doi:10.1093/ofid/ofy265 system, which is all easy to use. Visit http://www.dovepress.com/ testimonials.php to read real quotes from published authors.

\section{Dovepress}

